\title{
Virtual reality: What is the state of play in education?
}

\author{
Colin Macpherson \\ Central Queensland University
}

Mike Keppell

University of Melbourne

\section{Background}

The term 'virtual reality' (VR) is currently used to describe a range of computer-based systems in which a user can explore a hardware and software generated 'microworld' that bears some resemblance to reality. An early application of such systems was the flight simulator used to train pilots. However, it is in the area of hi-tech games that many of the more recent developments in this field have occurred. Typically, a user will wear a helmet containing either a small video screen positioned in front of each eye, or a device that projects images directly onto the user's retinas. She might also wear an elaborately wired glove that provides tactile feedback as she attempts to physically interact with the computergenerated visual environment. It was on devices and systems of this nature that our proposed investigation was to concentrate. Although this has remained the case, we have expanded our work to also include VR that mainly uses screen-based graphics - thus reflecting the expanded definition of VR (more of which later).

\section{Overall aim}

Our overall aim was to determine the nature and capabilities of VR devices and systems that have already been developed, and of those that are under development; and to investigate the educational and instructional uses to which these devices and systems are already being put and to which they may be put in the near future. In this regard it was not our intention to argue the case for VR in education - indeed, in some cases we would do quite the contrary - but to provide relevant 
information, and what we hoped would be enlightened discussion, so that educators in different situations could make up their own minds on the issues.

\section{Objectives}

In pursuing this aim we set ourselves the following objectives:

1) To clearly define what we mean by "virtual reality" (there is a range of definitions in common use).

2) To carry out a literature search both on-line (e.g., the WWW) and in paper-based publications to determine:

(a) the extent and technical capabilities of currently available devices and systems and,

(b) the extent of research and practice regarding the educational applications of such devices and systems.

3) To determine, through correspondence and by visiting appropriate centres, the nature and extent of research and development work on VR devices that is currently taking place.

4) To determine the current costs of available VR hardware and software and the development costs for new products.

5) To determine, at a general level, the nature of some of the technical problems currently faced in the field.

6) To reflect, evaluate and report on the current and near-future possibilities for applying VR systems to education.

\section{Information collecting methodology}

Being relative newcomers to VR, and knowing that our eventual audience would probably be even less aware of the field, we decided on a straightforward and systematic procedure to uncover the information we sought. Firstly we carried out a relatively extensive literature search. We say 'relatively' because a comprehensive search would have included an enormous amount of Internet searching where additions to the literature occur almost daily. We do believe, however, that our searches revealed enough data to see the 'big picture' together with some important detail. In an attempt to not stray too far from the educational 
focus of our investigation we began our search with the ERIC database using the simple keyword term 'virtual reality'. This revealed a modest 24 publications linking VR to an education context. This initial search also made clear the rate of change in the VR field. Some of the publications that were over five years old, although of historic interest, were clearly less relevant than more recent material - mainly because of technological achievements over a short span of years. Searches of other databases - mostly via the Internet - ultimately led to a compilation of relevant newsgroups, mailing lists, periodicals, books, and bibliographies. We examined and made a preliminary evaluation of all of these before carrying out the next step of securing more detailed information (i.e., full copies of books or journals or papers, and making contact with named sources). This process continued for the life of the project (i.e., through 1997), with new leads being followed and contacts being made, relevant information being recorded whenever appropriate.

During our literature search (or, more correctly, information search - the relevant material often not being purely in the form of literature) we came across information that we had been specifically looking for details of conferences that would be useful in our investigations, and contact details for educators who were heavily involved in VR. As a result, Mike Keppell attended the Virtual Reality Universe Conference and Exhibition in Santa Clara, California in April 1997, and Colin Macpherson visited the Virtual Reality and Education Laboratory at East Carolina University in June the same year. Both these visits were of great value in our investigations, giving us real contact with the technology and experts that hitherto we had only read about. Also in June 1997 the first world conference on 'VR in Education and Training' was held in the UK. Unfortunately, although we kept in close contact with the organiser and one keynote speaker, we were unable to attend.

As these information-gathering activities proceeded we began to map the boundaries of our overview of VR in education - each new piece of relevant material being integrated into the framework of headings and linkages. As the time for completion approached we again sifted through the data we had collected and re-evaluated the worth of many pieces. Through this process of searching, collecting, investigating, visiting and discussing with experts - as well as reflecting on what we had found - we were able to synthesise a lot of information into a relatively concise portrayal of the current state of VR technology and its present and possible future applications to education. 


\section{Results}

We believe objectives of the investigation have been met - in particular:

\section{1) Definitions}

The term 'virtual reality' has been defined in a number of ways since it was first coined - the focus being broadened in recent years. This has meant that many computer-based simulations that would have once been categorised differently are now included in the VR fold. Unfortunately, this broadening has tended to cause some confusion about what constitutes VR and what doesn't. Attempts have been made to clarify the situation by introducing qualifying terms such as 'deep immersion' which are tacked onto 'VR' in order to better describe the type of virtual reality being discussed.

Although this seems a logical approach, the possibility for further confusion was suggested when a fellow researcher with whom we met made the point that VR doesn't have to only apply to computergenerated images. What she meant was that participants must use their imaginations in order to make VR activities effective, and that the necessary imaginative processes can be stimulated in a number of ways that don't necessarily require hi-tech devices (e.g. reading a novel can lead to a type of VR experience). We decided that although this idea is of great importance, it was beyond the scope of the current investigation, but something to be addressed at another time.

What definition then? Hedberg and Alexander (1994) supply a sample of education-related definitions from the many in the literature, and then proceed to define a somewhat complex continuum where degree of immersion, fidelity of representation, and degree of active learner participation are defining dimensions. Wodaski (1995) attempts to side step the definition of VR by using a different term - 'artificial reality' - of which he considers VR to be a subset.

Drawing on these and other sources we have created our own straightforward definitions.

i) Virtual reality is a state produced in a person's mind that can, to varying degrees, occupy the person's awareness in a way similar to that of real environments.

ii) Virtual reality devices are devices that contribute to the creation of a virtual reality 
We realise that these definitions are very broad but we believe this degree of generality is necessary in order to encompass all of the simulations and tools that are now popularly described as examples of VR. However, there clearly needs to be a way of further defining the particular form of VR that is under discussion. As a result, we propose a number of qualifying terms as a means of focusing from the general to the more particular forms.

In terms of the relative overall effects produced by a package in its attempt to construct a virtual reality, the words low-end, medium, and high-end will be used. Examples of use, and the underlying range that is implied, will appear as our discussion proceeds. You will also notice that we use other qualifiers such as screen-based, three-dimensional screen-based, and non-immersive, immersive or fully immersive in order to focus on particular types and qualities of VR that are not only encompassed by our definition but also by popular usage of the term VR.

\section{2 a) Technical capabilities}

The technical capabilities of the many different sorts of VR devices on the market would take many pages to describe. If we limit the discussion to electronic, computer-based devices - the type most people associate with the term VR - then we can very briefly focus on a few categories that have seen significant developments in recent years. From the literature it is clear that the majority of this development work has taken place in the United States, and this is the country of origin of most commercially available VR technology. As a result, prices we mention are in US dollars. The technologies we will examine are:

\section{i) Head mounted devices (HMDs)}

These are devices that typically have a small (a couple of square centimetres) video screen placed a short distance in front of each eye - or in some cases miniature projection devices that project images directly onto each retina. Besides the quality of the images produced, another attribute of HMDs that has to be considered when evaluating their worth is the field-of-vision capability of the device. The average human horizontal field-of-vision is about $150^{\circ}$ but to produce an HMD with this capability requires curved screens and sophisticated lens systems which are very expensive - but such HMDs are available. The other important attribute of an HMD is its tracking capability. This refers to the device being able to detect the direction and speed of head movements, and for the rest of the VR system to react appropriately. For example, if the user 
suddenly lifts her head up in order to 'see' what's overhead then it is desirable that the system is able to refresh the screens accordingly in real time. Again, to do this properly is very expensive - not just for the sensing devices in the HMDs but also for the processing power of the hardware.

Costs and quality range from around US\$100 for a pair of LCD (Liquid Crystal Display) glasses that give poor-quality images that can only be seen whilst looking at a screen, to US\$95,000 for a true high-resolution HMD with wraparound viewing and sophisticated head tracking.

\section{ii) Gloves}

The concept of a glove containing various types of sensors or tactile feedback devices (or both) came early in the development of the VR field. The idea is to allow a user to interact with the visual VR environment using such a glove - which is connected to the computer hardware. The software coordinates the interaction of the gloved hand and the visual display. For example, the user may see an object in her virtual world and, with an appropriate glove, be able to pick up the object and place it somewhere else. Researchers in this area have also developed a number of tactile feedback devices to incorporate into such gloves. One particular type of these devices locks finger movements in the glove so that the user can 'feel' the solidity of the object - thus providing a form of feedback that bears some resemblance to the realworld experience. As with HMDs, the gloves that allow for smooth and easy interactions with virtual images are very expensive. The range of quality, and associated costs, is vast and typical of the field. The Mattel Power Glove, for example, when it was still being produced, was very basic in its operation but only cost approximately US $\$ 30$. The CyberGlove from Virtual Technologies, however, contains 22 sophisticated finger, palm, and wrist sensors - with each sensor costing around US\$2,000.

\section{iii) Motion sensing}

This branch of VR technology is already being used in the film and television industry. Basically, a human actor has white dots attached to a body suit at joint positions (e.g., wrists, ankles, elbows, knees, etc.) as well as on his or her head and face. A pair of video units detect the movement of these dots - each one being identified by the accompanying software - as the actor moves about on a stage that has a plain background. Similarly placed dots on a computer-generated 
virtual person are linked to those on the actor. With a lot of computing power and sophisticated software the virtual person can then be very realistically animated using various clips of the recorded movements of the actor. This process is known as performance animation.

In some applications the focus of the motion sensing is on the face of the actor - head, eye and mouth movements being mimicked on a speaking virtual character.

The purpose behind performance animation is to allow for the fast and accurate development of characters who are to inhabit a virtual world that is being created. Although this approach to animation has been used a lot in recent years for film production (e.g., the O. J. Simpson trial even had performance animations of what may have happened at the crime scene) the intention for virtual environments is to use combined clips of movements to give a virtual character some 'autonomy' - reacting differently according to circumstances. Again, for quality the cost is high. Ultra Trak from Polhemus uses a system that detects disturbances to a magnetic field, rather than the more typical video units. The cost of this system with its transmitter, computer and motion capture boards, and receivers averages around US $\$ 25,000$ (for 10 receivers). A videobased system with all the advanced hardware and software, such as that offered by Adaptive Optics, typically costs about US $\$ 100,000$.

\section{2 b) Education applications of VR}

Computer simulations for educational purposes have been used for many years - even before the advent of the microcomputer. Then, in the late 1970s and 1980s, simulations of this sort became commonplace in the classrooms and computer labs in most Western countries. These ranged in complexity, for example, from the simple simulation of the tossing of a coin in order to learn about binomial probability distributions, to the software recreation of an ecosystem in a lake where the interactions of various animal species were mimicked. Initially these simulations provided only textual and numeric output but as hardware became more powerful software was developed that allowed for graphical output.

Indeed, it is partly because of the rapid development of the graphics capabilities of computers that the term virtual reality is now also applied to software-animated, three-dimensional, screen-based graphics - some visual sequences today being indistinguishable from video footage of real-world events. The advances in HMD design have also been closely 
related to the rapid development of graphics hardware and software HMDs now having the capability of filling the user's whole visual field rather than being simply screen-based. Such devices and software would be described as high-end, immersive systems.

If we accept that three-dimensional, screen-based graphic simulations provide a form of VR (i.e., low-end) then we can justifiably state that VR is already being used in education. One example can be seen in the work of Veronica Pantelidis and Lawrence Auld, Co-directors of the Virtual Reality and Education Laboratory (VREL) at East Carolina University. Here, a software package called Virtus VR is used by primary school children to create the interior of a room or building, with walls, doors, windows and furniture being designed and edited and then placed in the appropriate part of the screen-based construction. Keyboardcontrolled movement through the three-dimensional representation is possible at any time. The aim of this activity is to promote children's ability to conceptualise in three-dimensional space, and to have fun and provide a sense of achievement whilst doing so.

Another example of the current use of VR in education is the Jason Project whereby school children can experience both 'telepresence' (the feeling of being in a location other than one's actual location) and 'teleoperation' (the remote operation of a device) (see McLellan 1995). In particular, this NASA program, which began in 1989, allows children in various locations around the United States to control the 'Telepresencecontrolled Remotely Operated underwater Vehicle' (TROV) as it explores the ocean depths of Antarctica, whilst viewing the real-time results of their actions on large video screens.

The Jason Project was designed to generate excitement in children about the study of science, mathematics, and technology at the school level (Franchi 1995). However, there is a growing number of uses of VR in higher education as well.

One such example is the Learning Sites Project where a team comprised of archaeologists, 3D Models and VR experts is building up graphic, audio and textual databases that enable users to explore a number of archaeological sites (so far, the Turkish site of Nemrud Dagi and temple B700 at Gebel Barkal in Egypt). Unlike relatively simple multimedia approaches, the use of VR in these cases allows the user to interact with virtual versions of the sites that have been created by the rendering of meticulously recorded data from the real site - the user deciding how 
that interaction should occur. For example, an obscure corner of a wall may be of interest to a particular student so, if she wants, she can zoom in on the area and investigate it in more detail. Connected to the Internet, these virtual environments will provide the potential for students to connect to databases and experts all over the world. The project team is currently led by Donald Sanders, an archaeologist at the Getty Institute. The intention is to make the products of this work available soon as an Internet-based teaching system, initially at East Carolina University and related school systems. The next stage in the program is to extend virtual reality into the realm of an active archaeological dig via telepresence and teleoperation techniques. Eban Gay, one of the team members who recently described the project, stated:

Virtual reality technology has only just become practical, but already it is being integrated into all aspects of archaeology, from research, through teaching, to tourism. This is a very exciting time to be involved with virtual technology (Gay, 1997).

Vocational and educational training (VET), including military uses in this field, is the area where a great deal of development work has taken place in using VR as an aid to learning. This is possibly because of the large sums of money that can be devoted to such activities by large commercial and defence organisations. The literature is replete with references to VET projects and commercially available VR products for training.

One such product is the Immersive Workbench developed by a company known as Fakespace. This product was on display at the 'Virtual Reality University $97^{\prime}$ conference in California last year. This particular technology allows a user with 3D glasses and specially-designed gloves to interact with three-dimensional objects suspended (virtually) above the Immersive Workbench. It is possible, for instance, to raise, lower and manipulate the different components of a car engine. By touching the thumb and forefinger together a user is able to highlight specific components of the engine and then move any of the pieces across the virtual benchtop. The Immersive Workbench allows this natural interaction with the computer-generated $3 \mathrm{D}$ imagery. The product is being used for scale-model manipulation in design projects and for medical visualisation. Fakespace suggests that the open table design allows collaborative workgroups to interact and work on projects at the same time. The workbench has been adopted by Silicon graphics, NASA Ames Research Center, and the Naval Research Laboratory in the US. 
This was one of the more intuitive VR applications seen at the conference because it allowed the use of a large number of natural manipulations - more or less confirming the following statement from the manufacturer: "Seeing the environment is just the first step in building virtual experiences. Control of virtual worlds is simplified by using natural motions that mirror the user's sense of real world activity" (Fakespace, 1997). Training applications are used by both Ford Motor Company in engineering and design and The Institute for Simulation and Training to train military hostage teams in how to best navigate around simulated building plans.

In terms of research into the educational applications of VR there is a lot going on. Pantelidis (1995) lists 300 articles, papers, Internet sites and materials in her bibliography of VR and education - many of these describing research projects. A feature of research that has this focus is that there is a great deal of activity at the school level (both primary and secondary) that uses relatively low-cost, low-end systems and devices, with the high-end systems being reserved for research into military and industrial training activities. Surprisingly, we had some difficulty in tracking down references to actual research and development projects at the higher-education level. One might assume that this was the case because the population of school students is larger than that of university students (i.e., a larger group of potential users), and because more money is available for high-level military and industrial training research than for research that focuses on university students. Another reason may be that the type of learning currently supported through VR environments is more aligned with the learning objectives of school and training programs than with that required at the tertiary level. Hedberg et al. (1994) gives a "Checklist for exploring virtual reality in education" in the form of 11 questions ranging from "to what extent is collaboration with peers possible and useful in the VR experience?" to "does the learner have to work with abstract relationships, manipulate data structures and mathematical functions?" They consider each question to suggest a strategy and context for research and development activity that VR is uniquely positioned to facilitate - if the right degree of sensory immersion and "representation fidelity" can be achieved.

\section{3) VR visits}

As part of this study two visits were made to VR facilities. Firstly, Mike Keppell attended the previously-mentioned 'Virtual Reality University 97' Conference at Santa Clara, California. As well as attending a number 
of paper presentations, he spent some time examining and using VR devices that were part of the commercial displays at the conference exhibition. His experiences, together with the information he was able to collect, were valuable resources for our investigation.

Secondly, Colin Macpherson acted on an invitation from the directors of the Virtual Reality and Education Laboratory (VREL) at East Carolina University (ECU), Dr Veronica Pantelidis and Dr Lawrence Auld, and visited their centre in June 1997. This experience was invaluable because Dr Macpherson was able to discuss issues he had formulated from his readings with two acknowledged pioneers who had spent a number of years investigating and promoting the possibilities of VR in education.

As well as editing and publishing a journal called 'VR in Schools' Drs Pantelidis and Auld are champions of the idea that low-cost, or even nocost (i.e., free) VR software can be used to promote useful learning particularly in younger students. An example of how they have implemented this idea was given earlier - their approach and pedagogical rationale being reminiscent of Papert's (1980) ideas about children creating 'microworlds' through the use of the LOGO computer language which he was instrumental in developing.

VREL is also responsible for the design of courses at ECU that use VR techniques (e.g., in chemistry) and for contributions to VR projects at a range of levels across the educational spectrum. The co-directors are also particularly well known because of their role in having VREL act as a clearing house for information from all around the world that focuses on VR in education.

\section{4) The cost of VR}

If one accepts that low-end VR includes three-dimensional, screenbased, animated graphics that can be viewed from various angles - the view taken at VREL - then VR can indeed be cheap. Assuming that users already have access to a moderately powerful computer, then the cost of the necessary software of this type can range from nothing (e.g., downloadable from the Web for free) for a package with fairly basic facilities such as an older version of 'Vetus VR', to something like 'Softimage 3D' from Microsoft which is very sophisticated and costs around US $\$ 8000$ - with a number of useful products priced within these extremes. The challenge for educational VR enthusiasts in this case is to find useful learning applications for this type of construction software. 
Towards the other end of the VR scale are the immersive devices, e.g., HMDs, gloves, body suits, etc. To have any of these provide anything like a reasonable degree of fidelity is expensive - and this is quite apart from extra costs associated with the necessary computing power, the VR development software, and the programming time necessary to create the 'realities' that are to be perceived through these devices. Wodaski (1995) discusses what VR investigators can expect to get for a range of 8 expenditures, starting at US $\$ 100$ for a cheap pair of LCD glasses that produce a flickering 3D image on a screen, to US\$100,000 for an HMD with quite sophisticated head-tracking and resolution, and perhaps even a glove and platform.

Clearly, investigators in the field need to first determine the type and quality of VR that will be necessary for their study, and then work out whether they can afford the hardware and software components necessary to fulfil their needs. As we have found, not all VR tools or devices are expensive but some are exceedingly so.

\section{5) Current technical problems}

The overriding problem facing the future development of VR technologies at present is associated with cost. To even gain reasonable quality with current systems is expensive. Organisations or groups that hope to push back the technological frontiers in the field will almost certainly need to be backed by very large sums of money. At present, much of the finance for such endeavours comes from the military - that sector's focus being mainly on the use of VR technologies for training and weapons control.

However, even with the most expensive and highest quality equipment there are still fundamental technical difficulties that appear to be - using present approaches - unsolvable. For example, haptic feedback (i.e., that sense of touch that lets you know, for instance, that you're holding a solid object) is currently provided with some gloves by an additional device that sits over the glove and, at appropriate times, restricts finger movement. The intention in this case is to provide an experience to the glove wearer that is something like the haptic feedback received when handling real objects. However, having your fingers restricted in their movement from above does not provide the same sensation as grasping a real object. (CyberGrasp is a device of this type. It fits over the CyberGlove mentioned earlier and very coarsely simulates normal haptic feedback. The cost of this add-on is approximately US\$40,000.) So 
far, there don't appear to be solutions to this problem of how to effectively simulate solidity in a virtual environment where nothing is solid.

Another technical problem that is restricting the pursuit of the dream of deep immersion VR is also associated with the sense of touch. In this case the problem focuses on the tactile feedback that we experience when we run our fingers, for example, over a surface. The current general approach to providing tactile feedback in VR systems is to have small devices in the fingertips of gloves. These devices vibrate at high frequencies when a virtual object is 'touched' by the fingers. However, when one considers the difference in sensation in the real world between running fingers over silk as compared to sandpaper, one can appreciate the difficulties faced by technology developers who want to provide a similar experience to VR users.

There is a range of other technical problems being faced in the VR field, some of them probably solvable but others of the same level of complexity as the haptic and tactile feedback challenges. The technology necessary to enhance the visual experience has developed very quickly over the last few years, and continues to develop at a fast pace. Audio simulation in VR has also moved quickly, but in regard to other senses there are clearly some very large and complicated barriers that may take some time to break through.

\section{6) The future of VR in education}

Three years ago there was an attempt by a number of high profile companies (e.g. Mattel) in the US and Japan to have VR become a consumer-driven technology - whereby developments in the field would focus on the desires of the masses (particularly in entertainment and edutainment). If this attempt had been successful then the cost of good quality VR devices and systems would almost certainly have dropped - perhaps to the point where immersive VR technology could be afforded by many educational researchers and educators in general. The reasons for the failure of this effort are numerous but a central issue was the poor quality of the products that were meant to 'kick-start' the sales and development process. As a result, the field is now back to being driven by the high-end technology required by the military and various industries. One likely outcome of this situation is that prices for good-quality equipment will remain generally high for a longer time. 
This means that education will, in general, have to focus on the nonimmersive, screen-based forms of VR. Although there will undoubtedly be an increase in the number of useful and innovative educational applications within this branch of VR, it is perhaps unfortunate that the exploration of how high-end VR might be applied to education will be relatively limited over the next few years.

Furthermore, many educators may doubt whether the use of low-end systems, that until a few years ago would not even have been called VR, is going to cause more than a ripple - let alone a revolution - in pedagogy and learning. Computer-based simulations have been used in education for some time now, and although the better quality products have undoubtedly been useful aids to learning, they have not conformed to the popular image of VR or of what it might have to offer education. (An example of this image is suggested by us in our presentation 'Is the elephant really there?' (Macpherson \& Keppell 1997) where we describe a hypothetical, fully-immersive VR system).

Undoubtedly, there will continue to be a place for the good-quality, lowend VR (i.e., screen-based graphical simulations) in education. This view is supported by the plethora of material that has appeared in the literature about such packages, and by the now well-established pedagogies that are meant to define their use. However, it is still at the high end where we suggest the 'big' possibilities may lie. Unfortunately, the costs in this realm are far too high at present for most educational research programs. Thorough studies of the implications of high-end VR for teachers and learners are, therefore, probably some time away and will probably only occur over a long period as costs slowly become more realistic and the advanced technology becomes more accessible. Even if high-end VR is a revolution waiting to happen in terms of education, the question of cost remains, i.e., will the benefits - assuming there are some - be worth the financial outlay? This, of course, is a question that applies to many other issues in education and can only be satisfactorily answered when the benefits are known and - more importantly - when the aims and objectives of educators are clearly established. Even then, given the range of values and perceptions in the field, it is doubtful whether general agreement is likely. But clearly, before educators can realistically expect to resolve such issues, as they apply to VR, a body of literature based on detailed, application-based research needs to evolve.

Moore (1995), in his conclusion about the state of VR in education, suggests that much of the discussion has centred on how VR might be used if the technology were more advanced, rather than focusing on 
how it can be used on the basis of what actually exists now. He goes on to state:

Current theory and methodology therefore is based on what will most likely be future fact, when the technologies associated with VR are able to do what they are being designed to do: to develop accurately-rendered worlds which can successfully create a complex illusion of cognitive presence. Once this is possible on a day-to-day basis, the concepts of learning through guided or self-guided experience in virtual worlds of variable verity may be attained. (p.101)

One possibility for further research that does not appear to have been explored in the VR literature is the development of virtual realities that are not based on computer technology - as alluded to earlier. This is an area in which Colin Macpherson carried out some preliminary investigations - in the past and in a different context - and one which may provide at least as much promise for education as the technologybased approach currently being used. This is certainly an area of research that should be pursued.

\section{References}

Fakespace (1997). Insights to Discovery. Fakespace, Mountain View, California.

Franchi, J. (1995). Virtual Reality: An Overview. ERIC Digest EDO-IR-95-5, pp.34.

Gay, E. (1997). Better Than the Real Thing? CyberEdge Journal, Issue 31, vol.6, no.3.

Hedberg, J. and Alexander, S. (1994). Virtual Reality in Education: Defining Researchable Issues. Educational Media International, 31(4), 214-220.

Macpherson, C. R. and Keppell, M. (1997). Is the Elephant Really There? - Virtual Reality in Education. Paper delivered at DDCE seminar, 3 October, Central Queensland University. http:/ / www.ddce.cqu.edu.au/ddce/confsem/

McLellan, H. (1995). Virtual field trips: The Jason project. VR World, 3(1), 49-50.

Moore, P. (1995). Learning and Teaching in Virtual Worlds: Implications of virtual reality for education. Australian Journal of Educational Technology, 11(2), 91-102. http: / / www.ascilite.org.au/ajet/ajet11/moore.html

Pantelidis, V. (1995). Virtual Reality and Education: Information Sources. Virtual Reality and Education Laboratory (VREL), East Carolina University, USA.

Papert, S. 1980, Mindstorms, The Harvester Press Ltd. Sussex, U.K.

Wodaski, R. 1995, Virtual Realist Madness!. 1996 Sams Publishing, Indianapolis, USA. 